Revista Arbitrada Interdisciplinaria KOINONIA

Año VI. Vol VI. N³. Edición Especial: Educación II. 2021

Hecho el depósito de Ley: FA2016000010 ISSN: 2542-3088

FUNDACIÓN KOINONIA (F.K). Santa Ana de Coro. Venezuela.

Milton Alejandro Guamán-Sigüenza; Carlos Marcelo Ávila-Mediavilla

http://dx.doi.org/10.35381/r.k.v6i3.1341

\title{
Aula invertida como estrategia metodológica activa
}

Flipped classroom as an active methodological strategy

\author{
Milton Alejandro Guamán-Sigüenza \\ maguamans35@grd.ucacue.edu.ec \\ Universidad Católica de Cuenca, Azogues \\ Ecuador \\ https://orcid.org/0000-0003-0266-6108 \\ Carlos Marcelo Ávila-Mediavilla \\ maguamans85@est.ucacue.edu.ec \\ Universidad Católica de Cuenca, Cuenca \\ Ecuador \\ https://orcid.org/0000-0002-2649-9634
}

Recepción: 10 de abril 2021

Revisado: 05 de mayo 2021

Aprobación: 30 de junio 2021

Publicación: 15 de julio 2021 
Revista Arbitrada Interdisciplinaria KOINONIA

Año VI. Vol VI. N³. Edición Especial: Educación II. 2021

Hecho el depósito de Ley: FA2016000010 ISSN: 2542-3088

FUNDACIÓN KOINONIA (F.K). Santa Ana de Coro. Venezuela.

\title{
RESUMEN
}

Se tiene por objetivo analizar el aula invertida como estrategia metodológica activa en la asignatura de Educación Física. Desde un contexto metodológico descriptivo con diseño no experimental. Es en este contexto, cuando el aula invertida se revela como una alternativa plenamente viable, para, en concordancia con las TICS, lograr un intercambio educativo activo en el cual el estudiante pase de ser un actor pasivo a un actor activo y, el docente vea potencializada su capacidad de transferencia de conocimientos. Es importante destacar la necesidad para el país de elevar el nivel educativo, a partir del uso de nuevas estrategias y herramientas de enseñanza capaces de potencializar el proceso de enseñanza - aprendizaje de educación física, rompiendo con esquemas y modelos pedagógicos tradicionales en los cuales no se verifica un intercambio bidireccional del conocimiento.

Descriptores: Método de enseñanza; enseñanza multimedia; aprendizaje active. (Palabras tomadas del Tesauro UNESCO).

\begin{abstract}
The objective is to analyze the flipped classroom as an active methodological strategy in the Physical Education subject. From a descriptive methodological context with a nonexperimental design. It is in this context, when the flipped classroom is revealed as a fully viable alternative, in order, in accordance with the ICTs, to achieve an active educational exchange in which the student goes from being a passive actor to an active actor and, the teacher sees potentiated their ability to transfer knowledge. It is important to highlight the need for the country to raise the educational level, based on the use of new teaching strategies and tools capable of enhancing the teaching-learning process of physical education, breaking with traditional pedagogical schemes and models in which there is no verifies a two-way exchange of knowledge.
\end{abstract}

Descriptors: Teaching methods; multimedia instruction; activity learning. (Words taken from the UNESCO Thesaurus). 


\section{INTRODUCCIÓN}

Los modelos educativos tradicionales mantienen las siguientes características o peculiaridades: el docente juega rol protagónico en el aula; se fomentan actividades que se orientan a la memorización; y, la retención y compresión de los conocimientos en el orden individual. Según la taxonomía propuesta Bloom (1964), estas habilidades corresponden a niveles bajos de trabajo cognitivo. Por ello, en la actualidad se aplican nuevas metodologías de enseñanza que permiten que el estudiante sea el protagonista de su propio proceso de aprendizaje, al mantener una participación activa, desarrollando sus habilidades comunicativas y de razonamiento (Cabrera-Solano \& Castillo-Cuesta, 2018); sin embargo, para su correcta aplicación es necesario un ajuste entre la docencia y los docentes (Gaete-Quezada, 2011)

Según (Campos-Gutiérrez et al. 20121), la Educación Física logra adherir el ejercicio físico a los estudiantes de los planteles educativos, es importante ofrecer un proceso de aprendizaje positivo sobre todo en la etapa adolescente y adulta, con el fin de influir sobre la vida del alumno, sin embargo, las limitadas horas disponibles para tratar esta importante materia, tan solo permiten mantener un reducido tiempo para las actividades motrices, por lo cual es imprescindible el uso de nuevas metodologías, a modo de estrategias innovadoras, para lograr eficiencia en la enseñanza. Por otro lado, la tecnología digital hace ya algunos años ha revolucionado la enseñanza como la conocíamos, incitando transformaciones que van desde las estrategias de enseñanzaaprendizaje hasta el currículo, tanto en el rol de los estudiantes como de los docentes (Pérez-Mateo-Subirà et al. 2014), en este sentido, las TIC proporcionan acceso instantáneo a la información, mediante el empleo de disimiles dispositivos con acceso a internet; por lo cual, es importante el correcto aprovechamiento de estas tecnologías disponibles, hacia todas las áreas de la educación, y la Educación Física no es la excepción (Zainuddin \& Halili, 2016).

En el proceso de inmersión en este nuevo mundo de la tecnología, surge como alternativa el modelo llamado "Aula Invertida", o "Flipped Classroom" (Lage et al., 2000), el mismo 
surge como contrapeso a la corriente pedagógica actual en educación física, esta nueva metodología propone dar la vuelta a la clase tradicional e invertir el orden en el proceso de aprendizaje (Collazos \& Mendoza, 2016).

El aula Invertida, es considerado un modelo de aprendizaje enseñanza de tipo semipresencial o mixto, ya que cuenta con una etapa presencial y otra virtual, a distancia. No es más que auxiliándonos de herramientas multimedia, realizar en casa lo que comúnmente se hace en el aula; y, lo que normalmente se hace en casa (como los deberes), se realiza en el aula, según refieren (Bergmann \& Sams, 2014). Cabe mencionar que el uso del método del Aula Invertida, podría aumentar la motivación de los alumnos, disminuye la frustración cuando el estudiante no puede resolver la tarea en casa, sin apoyo, contribuye a disminuir la deserción estudiantil, el docente se concentra en resolver problemas complejos; y, beneficia en la retroalimentación del grupo (Hew \& Lo, 2018).

Por lo expuesto, el objetivo principal del presente estudio es presentar la metodología del Aula Invertida como una posibilidad para su implementación en las clases de Educación Física de octavo año de educación básica de la Escuela "Gabriela Mistral", de la ciudad de Cuenca, Provincia del Azuay; partiendo de reconocer los recursos con los que cuenta el estudiantado, para la aplicación de esta modalidad. Además, esta metodología apoyará el uso de las tecnologías de la información y la comunicación y tratará de aprovechar las oportunidades que ofrece.

Uno de los primeros antecedentes que se conoce es el trabajo realizado por Walvoord y Johnson Anderson en el año 1998, mencionado por (Luque-Domínguez \& ParradoGallardo, 2019), quienes propusieron un modelo donde los estudiantes tenían que desarrollar contenidos previamente a la clase, para después, en la clase misma, fomentar la comprensión de los contenidos a través de un aprendizaje activo, esta comprensión pasaba por sintetizar, analizar y resolver problemas; y, con la finalidad de asegurar que los estudiantes lleven a cabo la preparación necesaria para el trabajo en el aula, se realizaban ensayos y cuestionarios antes de la clase. 
Por otro lado, según (Martínez et al., 2014) el término "aula invertida" fue acuñada por (Lage et al., 2016), para explicar las estrategias a utilizarse en clases de Economía; y años más tarde, fue utilizado por Bergmann y Sams, maestros de química, para realizar clases por videoconferencia con sus estudiantes. Mediante el uso de tecnologías de la información y la comunicación (TIC), el aula invertida es una nueva metodología de enseñanza y aprendizaje que permite a los estudiantes acceder de forma inmediata a nuevos contenidos en plataformas relevantes.

El aula invertida ha transformado ciertos procesos que estaban enlazados solo al aula, transfiriéndolos al contexto extraescolar. Este método, cambia totalmente la visión tradicional de clase: fuera del aula se realizarán esas actividades que tienen como fin la exposición y explicación de contenidos, por medio de herramientas tecnológicas como puede ser el vídeo o el podcast, o sencillamente internet. Así el tiempo escolar se dedica primordialmente a la realización de las actividades que efectivamente importan para el aprendizaje, como pueden ser los ejercicios prácticos, la resolución de dudas y problemas, los debates, los trabajos en pequeño o gran grupo, el aprendizaje por descubrimiento, la coevaluación y autoevaluación, etc.

Según (Guerrero, 2020), para que el aprendizaje invertido se considere un método de enseñanza en el aula, debe tener 4 pilares, resumidos en el acrónimo "FLIP", que significa: entorno flexible, cultura de aprendizaje, contenido dirigido y, anfitrión profesional. Este nuevo modelo de enseñanza no es tradicional, pues busca un aprendizaje eficaz y capaz entre los estudiantes, a través de herramientas digitales. Invierte el momento tradicional de la relación maestro-alumno, es decir, los maestros preparan los materiales del curso con anticipación, o graban sus propios temas dictados en clase, o compilan videos existentes en línea y luego distribuyen estos materiales a los estudiantes, de esta forma, pueden verlo en casa y venir al aula con conocimientos previos de temas relacionados.

A diferencia de otras disciplinas con mayores cargas teóricas, la integración de las TIC en el ámbito del deporte aún no ha jugado un papel, ya que, a primera vista, las TIC no 
son un elemento que se pueda relacionar con el deporte, considerando que el contenido a procesar está relacionado con juegos deportivos, deportes, expresión corporal, condición física básica, etc.; sin embargo, el actual enfoque integral otorgado a esta asignatura hace que su relación con las nuevas tecnologías sea cada vez más amplia y necesaria (Corrales, 2009). En concreto, en el proceso de buscar el mayor tiempo de ejercicio, es significativo aplicar la modalidad didáctica que garantiza el ejercicio físico de alto tiempo, que a su vez promueve el trabajo de diferentes tipos de contenidos (conceptuales, motrices, sociales, etc.) incluido en el plan de estudios en el ámbito de los deportes. Teniendo en cuenta sus características y posibilidades, el aula invertida, un método de enseñanza apoyado en las tecnologías de la información y la comunicación, se considera capaz de cubrir las múltiples necesidades de la educación física actual.

Por lo tanto, "invertir" en una clase va mucho más allá de la edición y distribución de materiales multimedia que los estudiantes deben ver en casa, es un enfoque integrado que combina la enseñanza directa con el constructivismo. Métodos y actitudes responsables y participación del alumno en el trabajo y los contenidos obtenidos (Tourón \& Santiago-Campión, 2013). Debido al auge que ha cobrado la tecnología en las vidas de los seres humanos y los beneficios que reporta en la educación por facilitar el aprendizaje y el desarrollo del estudiantado, se considera que es necesario aplicarlo porque no en la enseñanza de la educación física y es en lo que se basa este artículo.

\section{METODOLOGÍA}

La investigación fue de tipo descriptiva con diseño no experimental, para llevar a cabo esta investigación se establecieron como universo 28 alumnos de 8 vo grado de la Escuela "Gabriela Mistral", de la ciudad de Cuenca, aunque al final se presentaron únicamente 21, (7 estudiantes de género masculino y 14 de género femenino), de edades entre 12 y 14 años.

Se aplicó una encuesta conformada por 15 preguntas en total; 8 preguntas dirigidas a conocer el nivel de percepción que mantenían los estudiantes con la metodología actual 
en sus clases de Educación Física.; y, 7 preguntas encaminadas a conocer si los estudiantes tenían las herramientas y los conocimientos necesarios para la implementación del aula invertida. Para establecer la confiabilidad del instrumento utilizado, se realizó el cálculo del Alfa de Cronbach, obteniendo el siguiente resultado 0.782 para 8 preguntas, lo que significa que la confiabilidad es aceptable, y por lo tanto, los datos obtenidos pueden ser utilizados en el análisis del presente estudio.

\section{RESULTADOS}

En esta sección se brindarán los principales resultados obtenidos a partir de las preguntas planteadas durante el proceso de investigación. La información obtenida será analizada al final de cada cuadro.

\section{Tabla 1.}

Frecuencia en las evaluaciones realizadas por el profesor de Educación Física para verificar el progreso del estudiante.

\begin{tabular}{llrrrr}
\hline & Frecuencia & Porcentaje & $\begin{array}{c}\text { Porcentaje } \\
\text { válido }\end{array}$ & $\begin{array}{c}\text { Porcentaje } \\
\text { acumulado }\end{array}$ \\
\hline Válido & Nunca & 3 & 14,3 & 14,3 & 14,3 \\
& Algunas veces & 6 & 28,6 & 28,6 & 42,9 \\
& Frecuentemente & 5 & 23,8 & 23,8 & 66,7 \\
& Siempre & 7 & 33,3 & 33,3 & 100,0 \\
\cline { 2 - 5 } & Total & 21 & 100,0 & 100,0 & \\
\hline
\end{tabular}

Fuente: Encuesta.

Del total de estudiantes encuestados, el 33,33\% y el 23,81\% mencionó que los docentes realizan evaluaciones siempre y frecuentemente, respectivamente, para verificar su progreso; mientras que el $14,29 \%$ refieren que nunca son evaluados. 
Tabla 2.

Uso de las TIC por parte del profesor de Educación Física, para facilitar el proceso de aprendizaje.

\begin{tabular}{llllll}
\hline & Frecuencia & Porcentaje & $\begin{array}{l}\text { Porcentaje } \\
\text { válido }\end{array}$ & $\begin{array}{l}\text { Porcentaje } \\
\text { acumulado }\end{array}$ \\
\hline Válido & Nunca & 1 & 4,8 & 4,8 & 4,8 \\
& Algunas veces & 4 & 19,0 & 19,0 & 23,8 \\
& Frecuentemente & 2 & 9,5 & 9,5 & 33,3 \\
& Siempre & 14 & 66,7 & 66,7 & 100,0 \\
\cline { 2 - 5 } & Total & 21 & 100,0 & 100,0 & \\
\hline
\end{tabular}

Fuente: Encuesta.

Del total de estudiantes encuestados, el $66,7 \%$ mencionó que los docentes usan la tecnología en el proceso de aprendizaje, mientras que el 19\% dijo que usan estos métodos solo ocasionalmente.

Tabla 3.

Desarrollo de actividades que favorecen el trabajo en equipo, de forma virtual, por parte del profesor de Educación Física.

\begin{tabular}{llrrrr}
\hline & Frecuencia & Porcentaje & $\begin{array}{c}\text { Porcentaje } \\
\text { válido }\end{array}$ & $\begin{array}{r}\text { Porcentaje } \\
\text { acumulado }\end{array}$ \\
\hline Válido & Nunca & 2 & 9,5 & 9,5 & 9,5 \\
& Algunas veces & 4 & 19,0 & 19,0 & 28,6 \\
& Frecuentemente & 4 & 19,0 & 19,0 & 47,6 \\
& Siempre & 11 & 52,4 & 52,4 & 100,0 \\
\cline { 2 - 6 } & Total & 21 & 100,0 & 100,0 & \\
\hline
\end{tabular}

Fuente: Encuesta.

Del total de los estudiantes encuestados el 52,38\% afirman que el docente responsable de la asignatura fomenta la utilización de la tecnología en el desarrollo de las actividades en equipo mediante el empleo de las aulas virtuales, foros, video conferencias etc. Por otro lado, el 19,05\% refiere que frecuentemente esto sucede así. 
Tabla 4.

El estudiante posee computador, Tablet o celular en su hogar.

\begin{tabular}{llrrrr}
\hline & Frecuencia & $\begin{array}{c}\text { Porcentaj } \\
\text { e }\end{array}$ & $\begin{array}{c}\text { Porcentaje } \\
\text { válido }\end{array}$ & $\begin{array}{r}\text { Porcentaje } \\
\text { acumulado }\end{array}$ \\
\hline Válido & Algunas veces & 4 & 19,0 & 20,0 & 20,0 \\
& Siempre & 16 & 76,2 & 80,0 & 100,0 \\
& Total & 20 & 95,2 & 100,0 & \\
Perdidos & Sistema & 1 & 4,8 & & \\
Total & & 21 & 100,0 & & \\
\hline
\end{tabular}

Fuente: Encuesta.

Del total de los estudiantes encuestados el $76,2 \%$ poseen en su hogar algún medio tecnológico de los enunciados anteriormente, mientras que solo el $19 \%$ algunas veces poseen estos recursos.

\section{Tabla 5.}

Uso del estudiantado de plataformas web como Youtube, Facebook, Instagram, entre otros, en su proceso de aprendizaje.

\begin{tabular}{llrrrr}
\hline & & Frecuencia & Porcentaje & $\begin{array}{c}\text { Porcentaje } \\
\text { válido }\end{array}$ & $\begin{array}{r}\text { Porcentaje } \\
\text { acumulado }\end{array}$ \\
\hline Válido & Algunas veces & 4 & 19,0 & 20,0 & 20,0 \\
& Frecuentemente & 7 & 33,3 & 35,0 & 55,0 \\
& Siempre & 9 & 42,9 & 45,0 & 100,0 \\
& Total & 20 & 95,2 & 100,0 & \\
Perdidos & Sistema & 1 & 4,8 & & \\
\hline Total & & 21 & 100,0 & & \\
\hline
\end{tabular}

Fuente: Encuesta.

El gráfico que se presenta anteriormente muestra que $45 \%$ de los estudiantes siempre utilizan las plataformas web para visualizar información que ayuda a mejorar y a optimizar el proceso de aprendizaje, pero también en esta representación de los datos recogidos se observa que el solo el $35 \%$ frecuentemente las emplea. 


\section{Tabla 6.}

Uso de Office y PDF en la elaboración de tareas estudiantiles.

\begin{tabular}{llrrrr}
\hline & Frecuencia & Porcentaje & $\begin{array}{c}\text { Porcentaje } \\
\text { válido }\end{array}$ & $\begin{array}{r}\text { Porcentaje } \\
\text { acumulado }\end{array}$ \\
\hline Válido & Nunca & 2 & 9,5 & 10,0 & 10,0 \\
& Algunas veces & 4 & 19,0 & 20,0 & 30,0 \\
& Frecuentemente & 3 & 14,3 & 15,0 & 45,0 \\
& Siempre & 11 & 52,4 & 55,0 & 100,0 \\
& Total & 20 & 95,2 & 100,0 & \\
Perdidos & Sistema & 1 & 4,8 & & \\
\hline Total & & 21 & 100,0 & & \\
\hline
\end{tabular}

Fuente: Encuesta.

La tabla 3 evidencia que el 52, 4\% de los estudiantes encuestados siempre emplean estas herramientas ofimáticas para la realización de sus tareas docentes. Por otra parte, dicha tabla también muestra que el 19\% de los alumnos algunas veces emplean estas herramientas.

Mediante la aplicación de las encuestas se pudo visualizar que la mayoría de los alumnos, si bien tienen una buena percepción sobre el actual método de enseñanza, hay puntos que se pueden mejorar: la frecuencia en que son evaluados sus progresos en la materia de Educación Física; el uso de las TIC por parte del profesor, durante las clases; y, la motivación que brinda el docente a los alumnos para alcanzar los objetivos de aprendizaje.

Finalmente se vislumbró que, pese a que el $80 \%$ de los alumnos tienen recursos tecnológicos en sus hogares (computador, Tablet, celular e internet), no siempre los utilizan para la elaboración de sus trabajos académicos, e incluso, el 50\% de estudiantes consideran que la información que existe en el internet no es muy confiable. Además, alrededor de un $30 \%$ de alumnos, no conocen bien el manejo de las plataformas web, redes sociales ni paquetes utilitarios, siendo necesario que, para la aplicación de la presente propuesta, se realice una intervención educativa al estudiantado, para que este 
pueda conocer los beneficios del empleo de la tecnología en la enseñanza de Educación Física.

\section{PROPUESTA}

Una vez se determine que este grupo de alumnos cuenta con los recursos humanos y materiales para aplicar el método del aula invertida en Educación Física, se procede a enseñarles cómo hacerlo mediante el uso de tecnología educativa.

La forma de trabajo se construye a través de 4 ejes básicos que son:

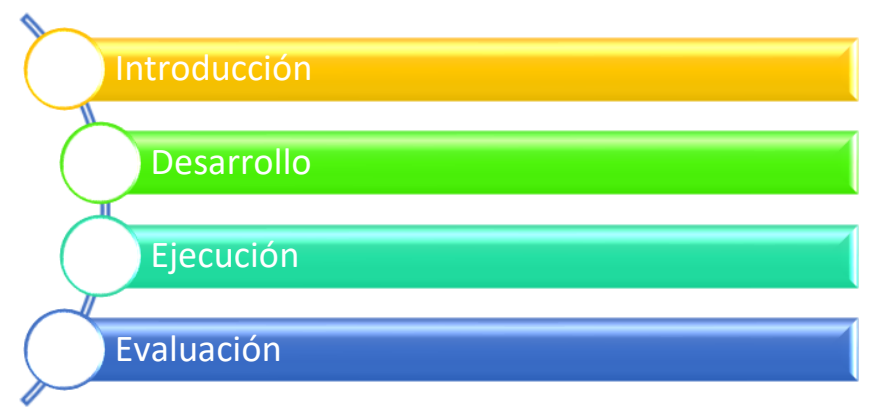

Figura 1. Desarrollo de la propuesta.

Elaboración: Los autores.

La aplicación se basa en que los miembros del grupo escuchen informes orales, luego se reúnan en grupos, utilicen técnicas de aprendizaje grupal para analizar la información y ponerla en práctica. El coordinador guiará las actividades propuestas y sugerirá un tiempo para cada actividad.

Introducción. - En este taller se mostrará cómo utilizar la tecnología antes, durante y después de las clases de Educación Física. Se abordarán objetivos y características de esta metodología y se compararán con la enseñanza tradicional para interiorizar las ventajas de este método. Además, se expondrán todos los pasos que se deben seguir de forma sistemática y explicativa, en la aplicación de dicha metodología, de una forma entendible y adecuada, según el nivel de conocimiento del grupo seleccionado. 
Desarrollo. - Durante las diferentes reuniones con el estudiantado, se pretende que los mismos sean capaces de entender y aplicar, de una manera efectiva, las TIC en el aprendizaje de la Educación Física.

Ejecución. - Para la ejecución de las sesiones, se han planificado las siguientes reuniones:

\section{Reunión 1}

Tema 1. Empleo de herramientas tecnológicas en el proceso de aprendizaje.

Contenido: Principales herramientas a utilizar para la aplicación de la metodología de aula inversa en Educación Física (laptops, celulares, tables).

Objetivos: Identificar dudas sobre el uso de esta tecnología en el proceso de aprendizaje de la asignatura.

Técnica: Exposición oral, Charla educativa, Dinámica de grupo, Demostración.

Materiales: Láminas, fotografías, plegables, power point, laptop, memoria de 2 GB.

Tiempo: 50 minutos.

\section{Reunión 2}

Tema 2. Búsqueda de información en las plataformas digitales.

Contenido: Ruta crítica para la búsqueda de información en la web. Plataformas seguras y no seguras para la obtención de la información.

Objetivo: Describir el método correcto para llegar a la información deseada y obtener información de calidad acerca del tema en cuestión e identificar posibles dudas acerca del tema.

Técnica: Exposición oral - Demostración, técnica participativa.

Materiales: Tablets, computador, power point, ilustraciones, internet.

Tiempo: 50 minutos. 
Revista Arbitrada Interdisciplinaria KOINONIA

Año VI. Vol VI. N³. Edición Especial: Educación II. 2021

Hecho el depósito de Ley: FA2016000010 ISSN: 2542-3088

FUNDACIÓN KOINONIA (F.K). Santa Ana de Coro. Venezuela.

Milton Alejandro Guamán-Sigüenza; Carlos Marcelo Ávila-Mediavilla

\section{Reunión 3}

Tema 3 Utilización del paquete Office en la realización de las tareas.

Contenido: Paquete Office en las tareas.

\section{Objetivos:}

- Identificar dudas respecto al temas.

- Determinar grado de dominio con que cuentan los estudiantes en este ámbito.

- Establecer pautas y reglas para el envío de los trabajos, utilizando estos medios.

Técnica: Tablet, computador, PowerPoint.

Tiempo: 50 minutos.

\section{Reunión 4.}

Objetivo: Reafirmar los conocimientos.

Método: Expositivo.

Tiempo: 50 minutos.

Evaluación: En esta sección se aclararán de manera global los problemas que surjan en el proceso. Primero, liderarán la discusión sobre si se han cumplido sus expectativas al inicio del ejercicio, y una ronda de preguntas y respuestas para aclarar posibles dudas. La reunión continuará probando la aplicación a través de presentaciones orales. La prueba permitirá evaluar el conocimiento de todas las preguntas obtenidas.

\section{CONCLUSIONES}

A través de esta investigación, se verificó que los estudiantes del octavo año de educación básica de la Escuela "Gabriela Mistral", tienen todas las condiciones para aplicar el enfoque de Aula Invertida a la Educación Física. Solo sobre esta base se elaboró la propuesta que permitirá abrir la mente de estos estudiantes y vincular el uso de la tecnología con la docencia, enfatizando sus ventajas.

El cuestionario aplicado permitió identificar que esta población tiene en sus manos herramientas digitales que hacen posible la aplicación del aula invertida como son las 
siguientes: la mayoría de los estudiantes cuenta con dispositivos que les permiten el acceso a Internet, pueden acceder a las redes a través de sus hogares y tienen bastante dominio en el uso de paquetes ofimáticos y plataformas digitales.

Las actividades desarrolladas en la propuesta de investigación van a permitir que el estudiante abra su mente y vincule el uso de la tecnología a el aprendizaje y la enseñanza de la asignatura de Educación Física.

Con esta propuesta de investigación ponen de manifiesto la importancia del aula invertida como herramienta y modelo pedagógico, capaz de trasladar parte del proceso de enseñanza-aprendizaje fuera del aula, de forma que se prioricen los procesos cognitivos significativos dentro del aula, lo que repercutirá positivamente en la materialización de aprendizajes significativos, a la vez que proporciona la oportunidad a los alumnos de ampliar los conocimientos adquiridos en un espacio de preferencia que motiva el aprendizaje de Educación Física.

El aula invertida proporciona un conjunto de pautas y pasos que potencian el aprendizaje de Educación Física, de manera individual, a los estudiantes de la Unidad Educativa Gabriela Mistral, de tal manera que les permite ampliar y profundizar los conocimientos adquiridos en el aula, a través del uso sistemático de las TIC, en sus propios espacios y preferencias, que varían en contenido pero que a la vez exigen el perfeccionamiento sistemático de la Educación Física como denominador común.

\section{FINANCIAMIENTO}

No monetario.

\section{AGRADECIMIENTO}

A la Jefatura de Posgrados de la Universidad Católica de Cuenca por permitir el desarrollo y fomento de la investigación. 
Revista Arbitrada Interdisciplinaria KOINONIA

Año VI. Vol VI. N³. Edición Especial: Educación II. 2021

Hecho el depósito de Ley: FA2016000010 ISSN: 2542-3088

FUNDACIÓN KOINONIA (F.K). Santa Ana de Coro. Venezuela.

Milton Alejandro Guamán-Sigüenza; Carlos Marcelo Ávila-Mediavilla

\section{REFERENCIAS CONSULTADAS}

Bergmann, J., \& Sams, A. (2014). Flipped learning: Maximizing face time. Learning \& Development. https://n9.cl/hhnu

Bloom, B. S. (1964). Taxonomy of educational objectives. https://n9.cl/aplnp

Cabrera-Solano, P., \& Castillo-Cuesta, L. (2018). Aplicación de la metodología de aula invertida en la enseñanza de la destreza de escritura en inglés como lengua extranjera. Un estudio de caso en Ecuador [Application of the flipped classroom methodology in the teaching of writing skills in English as a foreign language. A case study in Ecuador]. https://n9.cl/xe78u

Campos-Gutiérrez, L. M., Sellés-Pérez, S., García-Jaén, M., \& Ferriz-Valero, A. (2021). Aula invertida en educación física: aprendizaje, motivación y tiempo de práctica motriz. Revista Internacional De Medicina Y Ciencias De La Actividad Física Y Del Deporte, 21(81), 63-81. https://doi.org/10.15366/rimcafd2021.81.005

Collazos, C. A., \& Mendoza, J. (2016). Cómo aprovechar el "aprendizaje colaborativo" en el aula [How to take advantage of "collaborative learning" in the classroom]. Educación y Educadores, 9(2), 61-76.

Corrales, A. (2009). La integración de las tecnologías de la información y comunicación (TIC) en el Área [The integration of information and communication technologies (ICT) in the Area]. Hekademos: Revista Educativa Digital, 2(4), 45-56.

Gaete-Quezada, R. A. (2011). El juego de roles como estrategia de evaluación de aprendizajes universitarios [Role play as a strategy for evaluating university learning]. Educación y Educadores, 14(2), 289-307.

Guerrero, J. (comp.). (2020). Internacionalización del currículo y experiencias pedagógicas. Pertinencia y aprendizaje global en educación superior [Internationalization of the curriculum and pedagogical experiences. Relevance and global learning in higher education]. Bogotá D.C., Colombia: Corporación Universitaria Minuto de Dios. https://hdl.handle.net/10656/11585

Hew, K. F., \& Lo, C. K. (2018). Flipped classroom improves student learning in health professions education: a meta-analysis. BMC medical education, 18(1), 38. https://doi.org/10.1186/s12909-018-1144-z 
Lage, M., Platt, G., \& Treglia, M. (2000) Inverting the Classroom: A Gateway to Creating an Inclusive Learning Environment, The Journal of Economic Education, 31:1, 3043, DOI: $\underline{10.1080 / 00220480009596759}$

Luque-Domínguez, E. \& Parrado-Gallardo, E. (2019). La flipped classroom o clase invertida. https://n9.cl/ld848

Martínez, W., Esquivel, I., \& Castillo, J. M. (2014). Los Modelos Tecno-Educativos, revolucionando el aprendizaje del siglo XXI. In Aula invertida o modelo invertido de aprendizaje: Origen, sustento e implicaciones [The Techno-Educational Models, revolutionizing the learning of the XXI century. In Flipped classroom or flipped learning model: Origin, sustenance and implications]. https://n9.cl/ce4zd

Pérez-Mateo-Subirà, M., Romero-Carbonell, M., \& Romeu-Fontanillas, T. (2014). Collaborative construction of a project as a methodology for acquiring digital competences. [La construcción colaborativa de proyectos como metodología para adquirir competencias digitales]. Comunicar, 42, 15-24. https://doi.org/10.3916/C42-2014-01

Tourón, J., \& Santiago-Campión, R. (2013). Atención a la diversidad y desarrollo del talento en el aula. El modelo DT-PI y las tecnologías en la implantación de la flexibilidad curricular y el aprendizaje al propio ritmo [Attention to diversity and development of talent in the classroom. The DT-PI model and technologies in the implementation of curricular flexibility and self-paced learning]. Revista Española de Pedagogía, 279, 441-459.

Zainuddin, Z., \& Halili, S. H. (2016). Flipped Classroom Research and Trends from Different Fields of Study. The International Review of Research in Open and Distributed Learning, 17(3). https://doi.org/10.19173/irrodl.v17i3.2274

C2021 por los autores. Este artículo es de acceso abierto y distribuido según los términos y condiciones de la licencia Creative Commons Atribución-NoComercial-Compartirlgual 4.0 Internacional (CC BY-NC-SA 4.0)

(https://creativecommons.org/licenses/by-nc-sa/4.0/). 